\title{
Closeness and Distance Relations in Order of Magnitude Qualitative Reasoning via PDL*
}

\author{
A. Burrieza \\ Dept. Filosofía. Univ. de Málaga. Spain. \\ burrieza@uma.es
}

\author{
E. Muñoz-Velasco and M. Ojeda-Aciego \\ Dept. Matemática Aplicada. Univ. de Málaga. Spain. \\ \{emilio, aciego\}@etima.uma.es
}

\begin{abstract}
The syntax, semantics and an axiom system for an extension of Propositional Dynamic Logic (PDL) for order of magnitude qualitative reasoning which formalizes the concepts of closeness and distance is introduced in this paper. In doing this, we use some of the advantages of PDL: firstly, we exploit the possibility of constructing complex relations from simpler ones for defining the concept of closeness and other programming commands such as while ... do and repeat... until; secondly, we employ its theoretical support in order to show that the satisfiability problem is decidable. Moreover, the specific axioms of our logic have been obtained from the minimal set of formulas needed in our definition of qualitative sum of small, medium and large numbers. We also present some of the advantages of our approach on the basis of an example.
\end{abstract}

\section{Introduction}

The area of research within Artificial Intelligence that automates reasoning and problem solving about the physical world is called Qualitative Reasoning (QR). It creates nonnumerical descriptions of systems and their behaviour, preserving important behavioural properties and qualitative distinctions. Successful application areas include autonomous spacecraft support, failure analysis and on-board diagnosis of vehicle systems, automated generation of control software for photocopiers, conceptual knowledge capture in ecology, and intelligent aids for human learning. Order of magnitude reasoning is a part of QR which stratifies values according to some notion of scale (Raiman 1991; Dague 1993; Sánchez, Prats, and Piera 1996; Nayak 1994).

There are different approaches in the literature (Bennett et al. 2002; Wolter and Zakharyaschev 2002; Duckham et al. 2006) for using logic in $Q R$ that face the problem about the soundness of the reasoning supported by the formalism, and try to give some answers about the efficiency of its use. In particular, multimodal logics dealing with order of magnitude reasoning have been developed in (Burrieza and OjedaAciego 2005; Burrieza, Muñoz, and Ojeda-Aciego 2007) defining different qualitative relations (order of magnitude,

\footnotetext{
*Partially supported by projects TIN2006-15455-C03-01 and P06-FQM-02049.

Copyright (C) 2009, Association for the Advancement of Artificial Intelligence (www.aaai.org). All rights reserved.
}

negligibility, non-closeness, etc.) on the basis of qualitative classes obtained by dividing the real line in intervals (TravéMassuyès et al. 2005).

The syntax, semantics and an axiom system for a logic which formalizes the concepts of closeness and distance are introduced in this paper. To do this, we use the advantages of Propositional Dynamic Logic (Harel, Kozen, and Tiuryn 2000; Mirkowska and Salwicki 1987; Blackburn and Van Benthem 2007), mainly the possibility of constructing complex relations from simpler ones. Some recent applications of PDL in AI can be seen in (van Benthem, Eijck, and Kooi 2006; Heinemann 2007; Bugaychenko and Soloviev 2007; Bollig, Kuske, and Meinecke 2007). In our case, we define the concept of closeness as a program obtained by the union of the sum of classes representing zero, positive and negative small numbers. Moreover, we introduce some nominals in order to represent the different qualitative classes, for this reason we can say that our logic is a part of Combinatory PDL (Areces and ten Cate 2007; Passy and Tinchev 1991). This work continues the line of (Burrieza, Muñoz, and Ojeda-Aciego 2008) about using PDL in the framework of order of magnitude reasoning, however it introduces some differences, for example, here we use constants to represent the qualitative classes instead of the milestones which divide them, introducing an ordering only in the set of qualitative classes. This makes the approach more heavily founded on quantitativeness. Furthermore, this paper is an step forward in the formalization for two main reasons. Firstly, it gives a syntactic approach by presenting an axiom system where the specific axioms have been obtained from the minimal set of formulas needed in our definition of qualitative sum of small, medium and large numbers. Secondly, we have used the theoretical support of PDL in order to prove the decidability of the satisfiability problem in this logic.

The paper is organized as follows. In Section 2, the syntax and semantics of the proposed logic is introduced, together with an example of application of our logic. In Section 3, we give an axiom system for our logic and in Section 4 the decidability of the problem of satisfiability is proved. Finally, some conclusions and prospects of future work are presented in Section 5. 


\section{Syntax and Semantics}

In order to introduce the language of our logic, we consider a set of formulas $\Phi$ and a set of programs $\Pi$, which are defined recursively on disjoint sets $\Phi_{0}$ and $\Pi_{0}$, respectively. $\Phi_{0}$ is called the set of atomic formulas which can be thought as abstractions of properties of states. Similarly, $\Pi_{0}$ is called the set of atomic programs which are intended to represent basic instructions.

\section{Formulas:}

- $\Phi_{0}=\mathbb{V} \cup \mathbb{C}$, where $\mathbb{V}$ is a denumerable set of propositional variables and $\mathbb{C}=\{\mathrm{nl}, \mathrm{nm}, \mathrm{ns}, 0, \mathrm{ps}, \mathrm{pm}, \mathrm{pl}\}$. The elements of $\mathbb{C}$ are intended to represent, respectively the qualitative classes of "negative large", "negative medium", "negative small", "zero", "positive small", "positive medium", and "positive large" numbers.

- If $\varphi$ and $\psi$ are formulas and $a$ is a program, then $\varphi \rightarrow \psi$ (propositional implication), $\perp$ (propositional falsity) and $[a] \varphi$ (program neccesity) are also formulas.

\section{Programs:}

- $\Pi_{0}=\left\{+_{\star} \mid \star \in \mathbb{C}\right\}$.

- If $a$ and $b$ are programs and $\varphi$ is a formula, then $(a ; b)$ ("do $a$ followed by $b$ "), $a \cup b$ ("do either $a$ or $b$, nondeterministically"), $a^{*}$ ("repeat $a$ a nondeterministically chosen finite number of times") and $\varphi$ ? ("proceed if $\varphi$ is true, else fail") are also programs.

We now define the semantics of our logic. A model $\mathcal{M}$ is a tuple $(W, m)$, where $W$ is a non-empty set divided in 7 qualitative classes, chosen depending on the context (TravéMassuyès, Ironi, and Dague 2003), denoted for simplicity also by $\{\mathrm{nl}, \mathrm{nm}, \mathrm{ns}, 0, \mathrm{ps}, \mathrm{pm}, \mathrm{pl}\}$ and $m$ is a meaning function such that $m(p) \subseteq W$, for every propositional variable, $m(\star)=\star$, for every $\star \in \mathbb{C}$ and $m(a) \subseteq W \times W$, for all program $a$. Moreover, for every formula $\varphi$ and $\psi$ and for all programs $a, b$, we have:

- $m(\varphi \rightarrow \psi)=(W \backslash m(\varphi)) \cup m(\psi)$

- $m(\perp)=\varnothing$

- $m([a] \varphi)=\{w \in W:$ for all $v \in W$, if $(w, v) \in$ $m(a)$ then $v \in m(\varphi)\}$

- $m(a \cup b)=m(a) \cup m(b)$

- $m(a ; b)=m(a) ; m(b)$ (composition of relations $m(a)$ and $m(b))$

- $m\left(a^{*}\right)=m(a)^{*}$ (reflexive and transitive closure of relation $m(a))$.

- $m(\varphi$ ?) $=\{(w, w): w \in m(\varphi)\}$

The following properties are required for our atomic programs:

- $m\left(+_{\mathrm{ps}}\right)$ is a relation on $W$ such that:

1. $m(+\mathrm{ps})(\mathrm{nl}) \subseteq \mathrm{nl} \cup \mathrm{nm}$

2. $m(+\mathrm{ps})(\mathrm{nm}) \subseteq \mathrm{nm} \cup \mathrm{ns}$

3. $m(+\mathrm{ps})(\mathrm{ns}) \subseteq \mathrm{ns} \cup 0 \cup \mathrm{ps}$

4. $m(+\mathrm{ps})(\mathrm{ps}) \subseteq \mathrm{ps} \cup \mathrm{pm}$
5. $m\left(+_{\mathrm{ps}}\right)(\mathrm{pm}) \subseteq \mathrm{pm} \cup \mathrm{pl}$

6. $m\left(+_{\mathrm{ps}}\right)(\mathrm{pl}) \subseteq \mathrm{pl}$

- $m\left(+_{\mathrm{pm}}\right)$ is a relation on $W$ such that:

1. $m(+\mathrm{pm})(\mathrm{nl}) \subseteq \mathrm{nl} \cup \mathrm{nm} \cup \mathrm{ns}$

2. $m\left(+_{\mathrm{pm}}\right)(\mathrm{nm}) \subseteq \mathrm{nm} \cup \mathrm{ns} \cup 0 \cup \mathrm{ps} \cup \mathrm{pm}$

3. $m\left(+_{\mathrm{pm}}\right)(\mathrm{ns}) \subseteq \mathrm{ps} \cup \mathrm{pm}$

4. $m\left(+_{\mathrm{pm}}\right)(\mathrm{ps}) \subseteq \mathrm{pm} \cup \mathrm{pl}$

5. $m(+\mathrm{pm})(\mathrm{pm}) \subseteq \mathrm{pm} \cup \mathrm{pl}$

6. $m(+\mathrm{pm})(\mathrm{pl}) \subseteq \mathrm{pl}$

- $m(+\mathrm{pl})$ is a relation on $W$ such that:

1. $m\left(+_{\mathrm{pl}}\right)(\mathrm{nm}) \subseteq \mathrm{ps} \cup \mathrm{pm} \cup \mathrm{pl}$

2. $m\left(+{ }_{\mathrm{pl}}\right)(\mathrm{ns}) \subseteq \mathrm{pm} \cup \mathrm{pl}$

3. $m\left(+_{\mathrm{pl}}\right)(\mathrm{ps}) \subseteq \mathrm{pl}$

4. $m(+\mathrm{pl})(\mathrm{pm}) \subseteq \mathrm{pl}$

5. $m\left(+_{\mathrm{pl}}\right)(\mathrm{pl}) \subseteq \mathrm{pl}$

- $m\left(+_{\mathrm{ns}}\right), m\left(+_{\mathrm{nm}}\right)$ and $m\left(+_{\mathrm{nl}}\right)$ are given similarly and $m\left(+_{0}\right)$ is defined such that $m\left(+_{0}\right)=\{(w, w) \mid w \in W\}$.

Notice that the properties required for the specific atomic programs are intended to reflect intuitive properties of qualitative sum. For example, $m\left(+_{\mathrm{ps}}\right)(\mathrm{pl}) \subseteq \mathrm{pl}$ means that the sum of a positive small number plus a positive large number has to be a positive large number, and similarly for the rest of properties.

Given a model $\mathcal{M}=(W, m)$, a formula $\varphi$ is true in $u \in W$ whenever we have that $u \in m(\varphi)$. We say that $\varphi$ is satisfiable if there exists $u \in W$ such as $\varphi$ is true in $u$. Moreover, $\varphi$ is valid in a model $\mathcal{M}=(W, m)$ if $\varphi$ is true in all $u \in W$, that is, if $m(\varphi)=W$. Finally, $\varphi$ is valid if $\varphi$ is valid in all models.

The informal meaning of some of our connectives is given as follows:

- $\left\langle+_{\mathrm{ps}}\right\rangle \varphi$ is true in $u$ iff there exists $u^{\prime}$, obtained by adding a positive small number to $u$, such that $\varphi$ is true in $u^{\prime}$.

- $\langle\mathrm{nl} ?\rangle \varphi$ is true in $u$ iff $u$ is a negative large number and $\varphi$ is true in $u$.

- $\left\langle+_{\mathrm{ps}}^{*}\right\rangle \varphi$ is true in $u$ iff there exists $u^{\prime}$, obtained by adding a finitely many small positive numbers to $u$, such that $\varphi$ is true in $u^{\prime}$.

As stated above, one of the main advantages of using PDL is the possibility of constructing complex programs from basic ones. As a consequence, following the ideas presented in (Burrieza, Muñoz, and Ojeda-Aciego 2008), we can use our connectives in order to represent the relations of closeness and distance. Thus, for any formula $\varphi$, we define the modal connectives $[\mathrm{c}]$ and $[\mathrm{d}]$ as follows:

$$
\begin{gathered}
{[\mathrm{c}] \varphi=\left[+_{\mathrm{ns}} \cup+_{0} \cup+_{\mathrm{ps}}\right] \varphi} \\
{[\mathrm{d}] \varphi=\left[+_{\mathrm{nl}} \cup+_{\mathrm{pl}}\right] \varphi}
\end{gathered}
$$

The intuitive interpretation of the closeness relation is that $x$ is close to $y$ if, and only if, $y$ is obtained from $x$ by adding a small number. On the other hand, $x$ is distant from $y$ if and only if $y$ is obtained from $x$ by adding a large number. 
The following example was presented in (Burrieza, Muñoz, and Ojeda-Aciego 2007) for a multimodal logic. In this case, the use of PDL gives us many advantages, such as the possibility of expressing not only closeness and distance, but also some programming commands such as while...do and repeat... until.

Example Let us suppose that we want to specify the behaviour of a device to automatically control the temperature, for example, in a museum, subject to have some specific conditions. If we have to maintain the temperature close to some limit $T$, for practical purposes any value of the interval $[T-\epsilon, T+\epsilon]$ for small $\epsilon$ is admissible. This interval can be considered as ns $\cup 0 \cup p s$ in our approach. Moreover, assume that if the temperature is out of this interval (for example, because the number of people inside the museum is changing), it is necessary to put into operation either some heating or cooling system. We also assume that, when the normal system of cooling or heating is operating, a system to maintain the humidity is needed, and when the extra system is operating, we also need an extra system of humidification. As a consequence, the qualitative classes NL, NM, NS $\cup$ PS, PM and PL can be interpreted by the formulas: VERY_COLD, COLD, OK, HOT and VERY_HoT, respectively. We consider that program $+_{0}$ means that the system is off; moreover ${ }_{{ }_{p s}} \cup+_{\mathrm{pm}}$ and ${ }_{{ }_{\mathrm{pl}}}$, mean that a system for heating and extra heating are operating, respectively. Similarly we consider the meanings of programs $+{ }_{n m} \cup+{ }_{n s}$ and $+_{n l}$ for cooling and extra cooling operations, respectively. Some consequences of the previous specification are the following:

1. HOT $\rightarrow\left(\left[+_{\mathrm{pl}}\right]\right.$ VERY_HOT $\left.\wedge\left\langle\left(+_{\mathrm{nm}} \cup+_{\mathrm{ns}}\right)^{*}\right\rangle \mathrm{OK}\right)$

2. $\left[\left(\neg \mathrm{OK} \text { ?; }+_{\text {Sys }}\right)^{*}\right.$; OK?]OK, being $+_{\text {sys }}=+_{n l} \cup+{ }_{n m} \cup$ $+_{\mathrm{ns}} \cup+_{\mathrm{ps}} \cup+_{\mathrm{pm}} \cup+_{\mathrm{pl}}$

3. VERY_HOT $\rightarrow\left[\left(+_{\mathrm{nl}} ;\left(\neg \mathrm{OK} \text {; }+_{\mathrm{nl}}\right)^{*} ; \mathrm{OK} ?\right] \mathrm{OK}\right.$

4. $0 \rightarrow[\mathrm{c}] \mathrm{OK}$

5. OK $\rightarrow[d]($ VERY_COLD $\vee$ COLD $\vee$ HOT $\vee$ VERY_HOT $)$

We give now the intuitive meanings for the previous formulae.

- Formula 1 means that, if the temperature is hot and the extra heating system is put into operation, then the temperature will be very hot. Moreover, if the temperature is hot, the temperature becomes OK after finitely many applications of the cooling system.

- Formula 2 says that while the temperature is not OK, the system has to be operating, as a consequence, we will obtain the desired temperature.

- Formula 3 is interpreted as if the temperature is very hot, repeat the application of the extra cooling system until the temperature is $\mathrm{OK}$.

- Formula 4 means that every value close to the desired temperature is considered $\mathrm{OK}$.

- Formula 5 can be read in this way: if the temperature is $\mathrm{OK}$, for every distant value, the temperature will be either very cold or cold or hot or very hot.
If we assume that the system is more efficient (in terms of energy saving) if the temperature is close to the desired value $\mathrm{OK}$ and if the temperature is distant to these values, the system is wasting very much energy. the following formula must be true:

$$
\mathrm{OK} \rightarrow([\mathrm{c}] \text { efficient } \wedge[\mathrm{d}] \text { warning })
$$

This formula means that for every temperature close to $\mathrm{OK}$, the system is running efficiently and if the temperature is distant to $\mathrm{OK}$, the system is wasting very much energy.

\section{Axiom system}

We introduce here the axiom system for our logic.

\section{Axiom schemata for PDL:}

A1 All instances of tautologies of the propositional calculus.

$\mathbf{A 2}[a](\varphi \rightarrow \psi) \rightarrow([a] \varphi \rightarrow[a] \psi)$

$\mathbf{A 3}[a](\varphi \wedge \psi) \leftrightarrow([a] \varphi \wedge[a] \psi)$

$\mathbf{A 4}[a \cup b] \varphi \leftrightarrow([a] \varphi \vee[b] \varphi)$

A5 $[a ; b] \varphi \leftrightarrow[a][b] \varphi$

A6 $[\varphi ?] \psi \leftrightarrow(\varphi \rightarrow \psi)$

A7 $\left(\varphi \wedge[a]\left[a^{*}\right] \varphi\right) \leftrightarrow\left[a^{*}\right] \varphi$

A8 $\left(\varphi \wedge\left[a^{*}\right](\varphi \rightarrow[a] \varphi)\right) \rightarrow\left[a^{*}\right] \varphi$ (induction axiom)

We also consider as axioms the corresponding mirror images of K1-K4.

Axiom schemata for qualitative classes:

QE $n \mathrm{nl} \vee \mathrm{nm} \vee \mathrm{ns} \vee 0 \vee \mathrm{ps} \vee \mathrm{pm} \vee \mathrm{pl}$

QU $\star \rightarrow \neg \#$ for every $\star \in \mathbb{C}$ and $\# \in \mathbb{C}-\{\star\}$

QO1 $\mathrm{nl} \rightarrow\left\langle+_{\mathrm{ps}}^{*}\right\rangle \mathrm{nm}$

QO2 $\mathrm{nm} \rightarrow\left\langle+_{\mathrm{ps}}^{*}\right\rangle \mathrm{ns}$

QO3 ns $\rightarrow\left\langle+_{\mathrm{ps}}^{*}\right\rangle 0$

QO4 $0 \rightarrow\left\langle+_{\mathrm{ps}}^{*}\right\rangle$ ps

QO5 ps $\rightarrow\left\langle+_{\mathrm{ps}}^{*}\right\rangle \mathrm{pm}$

QO6 $\mathrm{pm} \rightarrow\left\langle+_{\mathrm{ps}}^{*}\right\rangle \mathrm{pl}$

Axiom schemata for specific programs:

PS1 $\mathrm{nl} \rightarrow\left[+_{\mathrm{ps}}\right](\mathrm{nl} \vee \mathrm{nm})$

PS2 $\mathrm{nm} \rightarrow\left[+_{\mathrm{ps}}\right](\mathrm{nm} \vee \mathrm{ns})$

PS3 ns $\rightarrow\left[+_{\mathrm{ps}}\right](\mathrm{ns} \vee 0 \vee \mathrm{ps})$

PS4 $p s \rightarrow\left[+_{p s}\right](p s \vee p m)$

PS5 $\mathrm{pm} \rightarrow\left[+_{\mathrm{ps}}\right](\mathrm{pm} \vee \mathrm{pl})$

PS6 $\mathrm{pl} \rightarrow\left[+_{\mathrm{ps}}\right] \mathrm{pl}$

PM1 $\mathrm{nl} \rightarrow\left[+_{\mathrm{pm}}\right](\mathrm{ns} \vee \mathrm{nm} \vee \mathrm{nl})$

PM2 $\mathrm{nm} \rightarrow\left[+_{\mathrm{pm}}\right](\mathrm{nm} \vee \mathrm{ns} \vee 0 \vee \mathrm{ps} \vee \mathrm{pm})$

PM3 ns $\rightarrow[{ } \mathrm{pm}](\mathrm{ps} \vee \mathrm{pm})$

PM4 ps $\rightarrow\left[+_{\mathrm{pm}}\right](\mathrm{pm} \vee \mathrm{pl})$

PM5 $\mathrm{pm} \rightarrow\left[{ }_{+\mathrm{pm}}\right](\mathrm{pm} \vee \mathrm{pl})$ 


$$
\begin{aligned}
& \text { PM6 } \mathrm{pl} \rightarrow\left[+_{\mathrm{pm}}\right] \mathrm{pl} \\
& \text { PL1 } \mathrm{nm} \rightarrow\left[+_{\mathrm{pl}}\right](\mathrm{ps} \vee \mathrm{pm} \vee \mathrm{pl}) \\
& \text { PL2 } \mathrm{ns} \rightarrow\left[+_{\mathrm{pl}}\right](\mathrm{pm} \vee \mathrm{pl}) \\
& \text { PL3 } \mathrm{ps} \rightarrow\left[+_{\mathrm{pl}}\right] \mathrm{pl} \\
& \text { PL4 } \mathrm{pm} \rightarrow\left[+_{\mathrm{pl}}\right] \mathrm{pl} \\
& \text { PL5 } \mathrm{pl} \rightarrow\left[+_{\mathrm{pl}}\right] \mathrm{pl}
\end{aligned}
$$

We also consider as axioms NS1... NS6; NM1... NM6 and NL1... NL5 by changing in the previous axioms every appearance of $p$ by $n$ and vice versa.

$$
\begin{aligned}
& \mathbf{Z 1}\left\langle+_{0}\right\rangle \varphi \rightarrow\left[+_{0}\right] \varphi \\
& \mathbf{Z 2}\left[+_{0}\right] \varphi \rightarrow \varphi
\end{aligned}
$$

\section{Inference Rules:}

(MP) $\varphi, \varphi \rightarrow \psi \vdash \psi$ (Modus Ponens)

(G) $\varphi \vdash[a] \varphi$ (generalization)

It is easy to establish that the following are derived rules of this system (Harel, Kozen, and Tiuryn 2000):

(I) $\varphi \rightarrow[a] \varphi \vdash \varphi \rightarrow\left[a^{*}\right] \varphi$ (invariance)

(D) $\varphi \rightarrow \psi \vdash[a] \varphi \rightarrow[a] \psi$ (distributivity)

Notice that axioms A1...A8 are classical for this type of logics. The rest ones have the following intuitive meaning:

- QE and QU mean the existence and uniqueness of the qualitative classes, respectively. Q01-Q06 represent the ordering of these qualitative classes.

- PS1-PS6, PM1-PM6 and PL1-PL5; the respective ones for negative numbers and 01-06 represent the desired properties of our atomic specific programs.

It is straightforward that all the previous axioms are valid formulas and that the inference rules preserve validity. For this reason, we can conclude that our system is sound, that is, every theorem is a valid formula.

\section{Decidability}

In order to obtain the decidability of the satisfiability problem, we prove the small model property. This property says that if a formula $\varphi$ is satisfiable, then it is satisfied in a model with no more than $2^{|\varphi|}$ elements, where $|\varphi|$ is the number of symbols of $\varphi$. This result can be obtained by the technique of filtrations used in modal logic (Blackburn, de Rijke, and Venema 2001). However, while in modal logic it is used the concept of subformula, in PDL we have to rely on the FisherLander Closure. All the results in this section can be proved in a standard way. For more details, see (Harel, Kozen, and Tiuryn 2000).

First of all, we define by simultaneous induction the following two functions, being $\Phi$ the set of formulas, $\Pi$ the set of programs of our logic and for every $\varphi, \psi \in \Phi, a, b \in \Pi$ :

$$
F L: \Phi \rightarrow 2^{\Phi} \quad F L^{\square}:\{[a] \varphi \mid a \in \Pi, \varphi \in \Phi\} \rightarrow 2^{\Phi}
$$

(a) $F L(p)=\{p\}$, for every propositional variable $p$.

(b) $F L(\star)=\star$, for all $\star \in \mathbb{C}$. (c) $F L(\varphi \rightarrow \psi)=\{\varphi \rightarrow \psi\} \cup F L(\varphi) \cup F L(\psi)$

(d) $F L(\perp)=\{\perp\}$

(e) $F L([a] \varphi)=F L^{\square}([a] \varphi) \cup F L(\varphi)$

(f) $F L^{\square}([a] \varphi)=\{[a] \varphi\}$, being $a$ an atomic program.

(g) $F L^{\square}([a \cup b] \varphi)=\{[a \cup b] \varphi\} \cup F L^{\square}([a] \varphi) \cup F L^{\square}([b] \varphi)$

(h) $F L^{\square}([a ; b] \varphi)=\{[a ; b] \varphi\} \cup F L^{\square}([a][b] \varphi) \cup F L^{\square}([b] \varphi)$

(i) $F L^{\square}\left(\left[a^{*}\right] \varphi\right)=\left\{\left[a^{*}\right] \varphi\right\} \cup F L^{\square}\left([a]\left[a^{*}\right] \varphi\right)$

(j) $F L^{\square}([\psi ?] \varphi)=\{[\psi ?] \varphi\} \cup F L(\psi)$

$F L(\varphi)$ is called the Fisher-Lander closure of formula $\varphi$.

The following result bounds the number of elements of $F L(\varphi)$, denoted by $|F L(\varphi)|$, in terms of $|\varphi|$. It is proved by simultaneous induction following the ideas presented in (Harel, Kozen, and Tiuryn 2000), taking into account our specific definition of $F L(\star)=\star$, for all $\star \in \mathbb{C}$, in the basis case of this induction.

\section{Lemma 1}

(a) For any formula $\varphi,|F L(\varphi)| \leq|\varphi|$.

(b) For any formula $[a] \varphi,\left|F L^{\square}([a] \varphi)\right| \leq|a|$, being $|a|$ the number of symbols of program $a$.

We now define the concept of filtration. First of all, given a formula $\varphi$ and a model $(W, m)$, we define the following equivalence relation on $W$ :

$$
u \equiv v \stackrel{\text { def }}{\Longleftrightarrow} \forall \psi \in F L(\varphi)[u \in m(\psi) \text { iff } v \in m(\psi)]
$$

The filtration structure $(\bar{W}, \bar{m})$ of $(W, m)$ by $F L(\varphi)$ is defined on the quotient set $W / \equiv$, denoted by $\bar{W}$, and the qualitative classes in $\bar{W}$ are defined, for every $\star \in \mathbb{C}$, by $\bar{\star}=\{\bar{u} \mid u \in \star\}$. Furthermore, the map $\bar{m}$ is defined as follows:

1. $\bar{m}(p)=\{\bar{u} \mid u \in m(p)\}$, for every propositional, variable $p$.

2. $\bar{m}(\star)=m(\star)=\star$, for all $\star \in \mathbb{C}$.

3. $\bar{m}(a)=\left\{(\bar{u}, \bar{v}) \quad \mid \quad \exists u^{\prime} \in \bar{u}\right.$ and $\exists v^{\prime} \in$ $\bar{v}$ such that $\left.\left(u^{\prime}, v^{\prime}\right) \in m(a)\right\}$, for every atomic program $a$.

$\bar{m}$ is extended inductively to compound propositions and programs as described previously in the definition of model.

The following two Lemmas are the key of this section and are proved following also the ideas presented in (Harel, Kozen, and Tiuryn 2000). To do this, we have to take into account that our definition of Fisher-Lander closure includes the qualitative classes and that the properties required in our models for atomic programs, such as $m(+\mathrm{ps})(\mathrm{nl}) \subseteq \mathrm{nl} \cup \mathrm{nm}$, are maintained in the filtration structure, as a direct consequence of our previous definitions.

Lemma $2(\bar{W}, \bar{m})$ is a finite model.

Lemma 3 (Filtration Lemma) Let $(W, m)$ be a model and $(\bar{W}, \bar{m})$ defined as previously from a formula $\varphi$. Consider $u, v \in W$.

1. For all $\psi \in F L(\varphi), u \in m(\psi)$ iff $\bar{u} \in \bar{m}(\psi)$. 
2. For all $[a] \psi \in F L(\varphi)$,

(a) if $(u, v) \in m(a)$ then $(\bar{u}, \bar{v}) \in \bar{m}(a)$;

(b) if $(\bar{u}, \bar{v}) \in \bar{m}(a)$ and $u \in m([a] \psi)$, then $v \in m(\psi)$.

As a consequence or the previous Lemmas, we can give the following result.

Theorem 1 (Small Model Theorem) Let $\varphi$ a satisfiable formula, then $\varphi$ is satisfied in a model with no more than $2^{|\varphi|}$ states.

Proof: If $\varphi$ is satisfiable, then there exists a model $(W, m)$ and $u \in W$ such that $u \in m(\varphi)$. Let us consider $F L(\varphi)$ the Fisher-Lander closure of $\varphi$ and the filtration model $(\bar{W}, \bar{m})$ of $(W, m)$ by $F L(\varphi)$ defined previously. From Lemma $2,(\bar{W}, \bar{m})$ is a finite model and by Lemma 3 (Filtration Lemma), we have that $\bar{u} \in \bar{m}(\varphi)$. As a consequence, $\varphi$ is satisfied in a finite model. Moreover, $\bar{W}$ has no more elements as the truth assignments to formulas in $F L(\varphi)$, which by Lemma 1 is at most $2^{|\varphi|}$.

\section{Conclusions and future work}

A PDL for order of magnitude reasoning has been introduced which deals with qualitative relations as closeness and distance. An axiom system for this logic has been defined by including as axioms the formulas which express syntactically the needed properties. Moreover, we have shown the decidability of the satisfiability problem of our logic.

As a future work, we are working in the proof of completeness of the given axiom system and the study of complexity of the satisfiability problem. Moreover, we are trying to extend this approach for more relations such as a linear order and negligibility, by maintaining decidability and completeness. Finally, we have planned to give a relational proof system based on dual tableaux for this logic in the line of (Burrieza, Ojeda-Aciego, and Orłowska 2006; Golińska-Pilarek and Muñoz Velasco 2009).

\section{References}

Areces, C., and ten Cate, B. 2007. Hybrid logics. In Handbook of Modal Logic, volume 3 of Studies in Logic and Practical Reasoning. Elsevier. 821-868.

Bennett, B.; Cohn, A.; Wolter, F.; and Zakharyaschev, M. 2002. Multi-dimensional modal logic as a framework for spatio-temporal reasoning. Appl. Intellig. 17(3):239 - 251. Blackburn, P., and van Benthem, J. 2007. Modal logic: A semantic perspective. In Handbook of Modal Logic, volume 3 of Studies in Logic and Practical Reasoning. Elsevier. 58-61.

Blackburn, P.; de Rijke, M.; and Venema, Y. 2001. Modal Logic. Cambridge University Press.

Bollig, B.; Kuske, D.; and Meinecke, I. 2007. Propositional dynamic logic for message-passing systems. Lecture Notes in Computer Science 4855:303-315.

Bugaychenko, D., and Soloviev, I. 2007. MASL: A logic for the specification of multiagent real-time systems. Lecture Notes in Computer Science 4696:183-192.
Burrieza, A., and Ojeda-Aciego, M. 2005. A multimodal logic approach to order of magnitude qualitative reasoning with comparability and negligibility relations. Fundamenta Informaticae 68:21-46, 2005.

Burrieza, A.; Muñoz, E.; and Ojeda-Aciego, M. 2007. A logic for order of magnitude reasoning with negligibility, non-closeness and distance. Lect. Notes in Artificial Intelligence 4788:210-219.

Burrieza, A.; Muñoz, E.; and Ojeda-Aciego, M. 2008. A propositional dynamic logic approach for order-ofmagnitude reasoning. Lect. Notes in Artificial Intelligence 5290:11-20.

Burrieza, A.; Ojeda-Aciego, M.; and Orłowska, E. 2006. Relational approach to order of magnitude reasoning. Lect. Notes in Computer Science 4342:105-124.

Dague, P. 1993. Symbolic reasoning with relative orders of magnitude. In Proc. 13th Intl. Joint Conference on Artificial Intelligence, 1509-1515. Morgan Kaufmann.

Duckham, M.; Lingham, J.; Mason, K.; and Worboys, M. 2006. Qualitative reasoning about consistency in geographic information. Inform. Sci. 176(6):601-627.

Golińska-Pilarek, J., and Muñoz Velasco, E. 2009. Relational approach for a logic for order of magnitude qualitative reasoning with negligibility, non-closeness and distance. Intl Journal of Computer Mathematics. To appear.

Harel, D.; Kozen, D.; and Tiuryn, J. 2000. Dynamic logic. MIT Press.

Heinemann, B. 2007. A PDL-like logic of knowledge acquisition. Lect Notes in Computer Science 4649:146-157.

Mirkowska, C., and Salwicki, A. 1987. Algorithmic Logic. Kluwer Academic Publishers.

Nayak, P. 1994. Causal approximations. Artificial Intelligence 70:277-334.

Passy, S., and Tinchev, T. 1991. An essay in combinatory dynamic logic. Inform. and Computation 93(2):263 - 332.

Raiman, O. 1991. Order of magnitude reasoning. Artificial Intelligence 51:11-38.

Sánchez, M.; Prats, F.; and Piera, N. 1996. Una formalización de relaciones de comparabilidad en modelos cualitativos. Boletín de la AEPIA (Bulletin of the Spanish Association for AI) 6:15-22.

Travé-Massuyès, L.; Prats, F.; Sánchez, M.; and Agell, N. 2005. Relative and absolute order-of-magnitude models unified. Annals of Math. and Artif. Intellig. 45:323-341.

Travé-Massuyès, L.; Ironi, L.; and Dague, P. 2003. Mathematical foundations of qualitative reasoning. AI Magazine 24(4):91-106.

van Benthem, J.; Eijck, J.; and Kooi, B. 2006. Logics of communication and change. Information and Computation 204(11):1620-1662.

Wolter, F., and Zakharyaschev, M. 2002. Qualitative spatio-temporal representation and reasoning: a computational perspective. In Lakemeyer, G., and Nebel, B., eds., Exploring Artificial Intelligence in the New Millenium. Morgan Kaufmann. 